\title{
Leg-ulcer care in the community, before and after implementation of an evidence-based service
}

\author{
Margaret B. Harrison, Ian D. Graham, Karen Lorimer, Elaine Friedberg, Tadeusz Pierscianowski, \\ Tim Brandys
}

Abstract

Background: Leg ulcers usually occur in older patients, a growing population for which increasing health care resources are required. Treatment is mainly provided in patients' homes; however, patients often receive poorly integrated services in multiple settings. We report the results of a prospective study of a community-based care strategy for leg ulcers.

Methods: International practice recommendations and guidelines were adapted to make a new clinical protocol. The new model, for a dedicated service staffed by specially trained registered nurses, established initial and ongoing assessment time frames and provided enhanced linkages to medical specialists. Data were collected for 1 year before and after implementation; outcome measures included 3-month healing rates, quality of life and resource usage.

Results: Three-month healing rates more than doubled between the year before implementation (23\% [18/78]) and the year afterward $(56 \%$ [100/180]). The number of nursing visits per case declined, from a median of 37 to 25 ( $p=0.041)$; the median supply cost per case was reduced from $\$ 1923$ to $\$ 406$ ( $p$ $=0.005$ ).

Interpretation: Reorganization of care for people with leg ulcers was associated with improved healing and a more efficient use of nursing visits.

CMAJ 2005;172(11):1447-52

A lthough not always recognized as a pressing health care problem, leg ulcers are a common, complex, and costly condition. International studies have shown that their occurrence increases with age.$^{1-5}$ Chronic ulcers are an ongoing burden to patients and to the health care system. ${ }^{6-10}$ Patients tend to receive poorly integrated services in multiple settings.

The Ottawa Community Care Access Centre, an eastern Ontario home-care authority, observed a pattern of yearly increases in the resources required to care for people with chronic wounds. Researchers from Queen's University, Ottawa University and 3 nursing agencies undertook a comprehensive regional needs assessment to understand the population and the care environment. They documented an estimated prevalence of 1.8 cases of leg ulcer per 1000 population, an incidence comparable to rates reported from other countries. ${ }^{11}$
Profile information revealed a population complex in terms of health problems and care challenges. Most patients were over 65 years in age; nearly three-quarters had 3 or more other conditions. Over two-thirds had experienced leg ulcers for many months. Half of the affected population had a leg-ulcer history spanning $5-10$ years; a third, exceeding 10 years. Our 4-week costing study ${ }^{12}$ estimated that 192 people receiving care would annually consume \$1 million in nursing care services and \$260 000 in wound-care supplies.

Home care nurses and family physicians had varying levels of confidence in managing patients with leg ulcers. ${ }^{13,14}$ In general, they were unaware of the relative effectiveness of compression therapy for venous leg ulcers, i.e., that 1 of 6 patients so treated would heal $(95 \%$ confidence interval [CI] 4-18). ${ }^{15,16}$ Practice audits indicated that assessments were not standardized: ultrasound readings of the ankle brachial pressure index (measured with a handheld Doppler) to rule out arterial disease were not routine; serial measurements of ulcers were carried out inconsistently; and compression bandaging, the standard of care for venous ulcers, was underutilized and yet also occasionally applied inappropriately to ulcers with arterial involvement. ${ }^{17}$

The researchers and organizations involved collaborated and fed information back and forth, generating regional data which, when combined with available external evidence, provided information appropriate to the local community. Regional decision-makers agreed to a redesign of the delivery of care to these patients, based on that information. This involved changing to a nurse-led service providing clinical care in accordance with a set of evidencebased guidelines. ${ }^{18-20}$ The objective of our study was to determine and compare the health outcomes and efficiencies of the former and new services.

\section{Methods}

We decided to study a mixed urban-rural population served by a single, large home-care authority; the region chosen was populated by about 1 million people. Individuals who spoke English or French and were newly referred for home-care management of their leg ulcer(s) were eligible to participate. The study inclusion criteria specified an ulcer below the knee, without arte- 
rial involvement; i.e., an open wound of the lower limb that resists healing, caused by venous or mixed venous-arterial disease. Community care was provided in the home or at either of 2 clinics staffed by nurses.

The old and new service models were evaluated prospectively with a "pre-/postimplementation" study design (Fig. 1) approved by the Ottawa Health Research Institute's Research Ethics Board. The service system already in place required changes both organizational and clinical. Organizational changes involved switching to a primary nursing delivery model: ${ }^{21}$ a dedicated service led and staffed entirely by registered nurses, with new provisions for physician referral and consultation. Each patient was assigned a primary nurse at admission, who was supported by a limited number of secondary nurses so that care could be provided 7 days per week.

Community nurses received training in how to assess leg ulcers and provide comprehensive care during a 12-month, in- depth distance education course (Thames Valley University, UK) that included hands-on master classes. Those who successfully completed the course received a university certificate and became primary nurses in the new service. Training for secondary nurses included an extensive orientation and a "buddy system" to increase expertise and create a critical mass of appropriately prepared practitioners.

Clinical changes entailed adopting an evidence-informed protocol developed by an interdisciplinary task force, whose members made use of the Practice Guideline Evaluation and Adaptation Cycle. ${ }^{22,23}$ International guidelines were evaluated not only by content analysis of their recommendations but also by means of a validated guideline appraisal instrument. The task force compared guidelines for content and methodological quality, and practitioners and external experts provided feedback to help identify the recommendations most appropriate to the region. The result was

Problems:

- Increasing numbers of patients with leg ulcers

- Rising costs of care

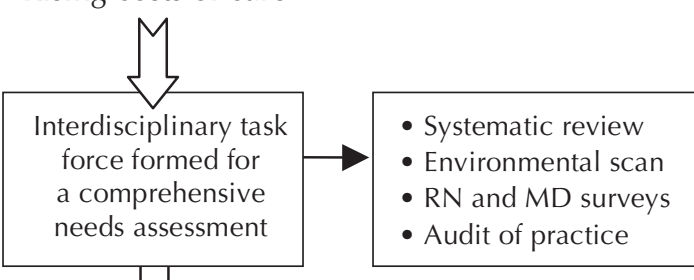

Findings:

- 1.8 of 1000 people have leg ulcers

- Many RNs or RPNs are needed per patient

- Referrals are made by MDs

- There is wide variation in care

- There are no standardized forms or measurements

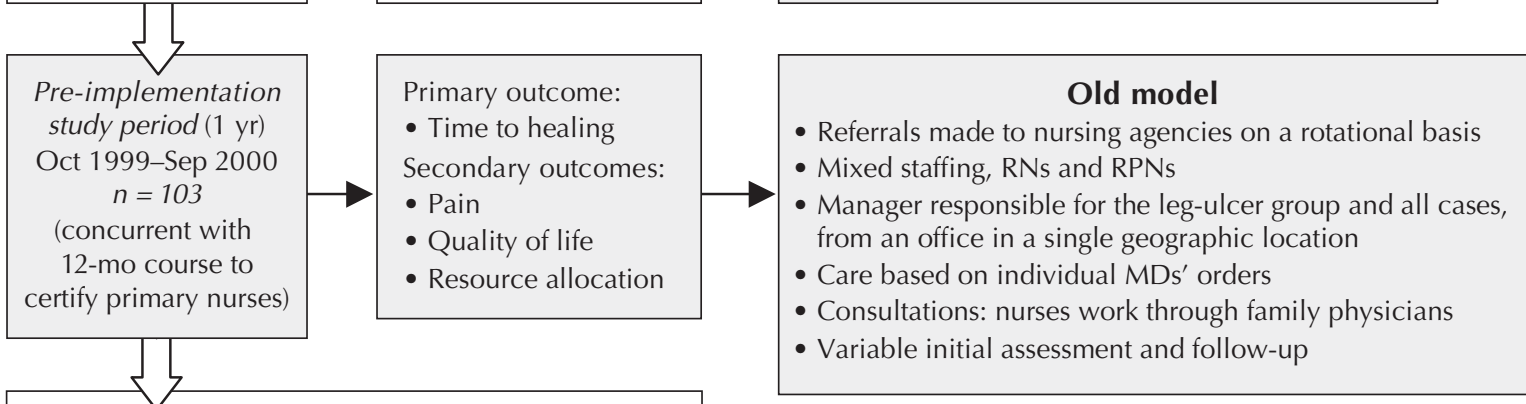

Washout period (1 mo) Oct 2000

- New service established No data collected

- Nurse orientations held

- New clinical protocol for leg ulcers introduced

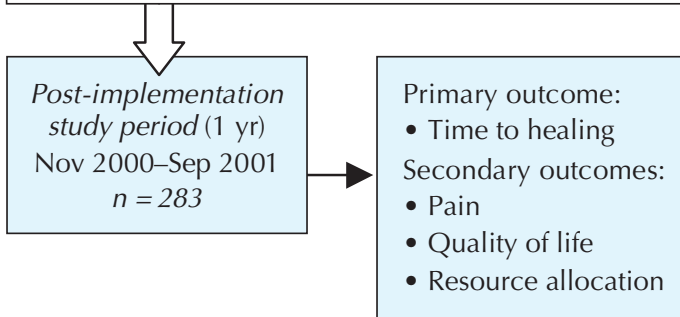

\section{New model}

- Regional service for leg ulcers centralized to 1 agency

- All services provided by RNs trained in leg-ulcer assessment and care

- Primary nurse service with team

- Team reports to a clinical leader who is responsible for orientation, continuing education and quality control as well as providing care

- Care protocol is evidence-based; individual orders by MDs are the exception rather than the rule

- Consultations: streamlined links to specialists for referral, notification to family physicians

- Standardized comprehensive, initial assessment (1-1.5 h) follow-up every 3 mo for $1 \mathrm{yr}$

Fig. 1. Flowchart indicating structure and time frame of study. RPNs = registered practical nurses. 
a local guideline, the Ottawa Carleton Community Care Leg Ulcer Protocol. ${ }^{19,20}$

According to the protocol, a primary nurse spends 1-1.5 hours doing an initial comprehensive assessment, using a standardized approach that includes Doppler measurement of the the ankle brachial pressure index. If the ulcer is of venous or venous-arterial origin and compression is deemed appropriate, the primary therapy of choice is compression bandaging. Serial measurements of the ulcer are part of the care routine, with full reassessment monthly; if no progress is noted, the patient is referred to a medical specialist. Once discharged, reassessment continues every 3 months until 1 year after admission, to support self-management, minimize recurrences and identify any recurrences early.

In both study periods, attending nurses were provided with information sheets to give to potential participants. After answering any questions from patients or accompanying caregivers, the nurses obtained written consent from those who agreed to join the study. If a patient was cognitively impaired, the information was given to a caregiver (if available), and permission was requested for the collection of clinical data only.

Data were collected at admission and again 3 months afterward. Profile information (demographic data, including information on patients' circumstances of living, and clinical data including comorbidities, previous leg ulcers and other medical history) was gathered through audit of health records. The study outcomes sought were the same for both study periods: the primary outcome was the proportion of patients whose leg ulcers had healed within 3 months of admission. Secondary outcomes included pain (as estimated with the Short Form McGill Pain Questionnaire, ${ }^{24}$ health-related quality of life (HRQL, measured with the Medical Outcomes Study 12-Item Short-Form Health Survey [SF-12] $)^{25}$ and resource utilization (e.g., nursing visits, and compression bandages and other supplies used). Study participants had the option of completing the SF-12 forms themselves or partaking in a structured interview.

Clinical outcomes were assessed by the attending nurses on regularly scheduled visits and recorded on a standardized form. When healing was successful and a patient was no longer receiving care, a nurse collected data during a special visit funded by the research study. Research staff collected data on resource utilization from the nursing agency involved, home-care health records and administrative databases.

Descriptive statistics were calculated to report clinical and resource utilization data. The $t$ test was used to evaluate differences between continuous variables (age, HRQL scores, ulcer size and persistence) and the $\chi^{2}$ test to compare categorical variables (e.g., Margolis prognostic indicators). ${ }^{26,27}$ Where an initial analysis indicated that differences were not normally distributed,

Table 1: Baseline characteristics of patient groups before and after implementation of the new model of care for leg ulcers

\begin{tabular}{|c|c|c|c|}
\hline \multirow[b]{2}{*}{ Characteristic } & \multicolumn{2}{|c|}{ Care; no. $(\%)$ of patients* } & \multirow[b]{2}{*}{$p$ value } \\
\hline & $\begin{array}{l}\text { Old model } \\
\quad n=78\end{array}$ & $\begin{array}{l}\text { New model } \\
n=180\end{array}$ & \\
\hline Female & $52(66.7)$ & $108(60.0)$ & 0.31 \\
\hline Age, yr, mean (SD) & $72.7(13.8)$ & $73.4(13.7)$ & 0.69 \\
\hline English-speaking & $57(74.0)$ & $48(82.2)$ & 0.28 \\
\hline Living alone & $31(39.7)$ & $74(41.1)$ & 0.84 \\
\hline Independently mobile & $44(56.4)$ & $102(57.3)$ & 0.93 \\
\hline History of ulceration & $37(47.4)$ & $94(52.8)$ & 0.43 \\
\hline $\begin{array}{l}\text { Previous episodes of leg ulcer, } \\
\text { mean no. (SD) }\end{array}$ & $2.7 \quad(1.9)$ & $3.1 \quad(3.9)$ & 0.52 \\
\hline \multicolumn{4}{|l|}{ Cause of leg ulcer } \\
\hline Venous disease & $57(73.1)$ & 109 (60.9) & 0.05 \\
\hline Mixed venous and arterial & $21(26.9)$ & $71(39.4)$ & \\
\hline Ulcer size, $\mathrm{cm}^{2}$, mean (SD) & $6.9(21.4)$ & $10.9(35.4)$ & 0.38 \\
\hline $\begin{array}{l}\text { Duration of ulcer at initial assessment, } \\
\text { wk, mean (SD) }\end{array}$ & 40.1 (98.5) & 34.4 (98.6) & 0.68 \\
\hline Ulcers $<5 \mathrm{~cm}^{2}$ & $n=71$ & $n=179$ & \\
\hline Present for $<6 \mathrm{mo}$ & $40(56.3)$ & $110(61.5)$ & 0.15 \\
\hline Present for $>6 \mathrm{mo}$ & $13(18.3)$ & $17 \quad(9.5)$ & \\
\hline Ulcers $>5 \mathrm{~cm}^{2}$ & $n=71$ & $n=179$ & \\
\hline Present for $<6 \mathrm{mo}$ & $15(21.1)$ & 35 (19.6) & \\
\hline Present for $>6 \mathrm{mo}$ & $3 \quad(4.2)$ & $17 \quad(9.5)$ & \\
\hline SF-12 scores, mean (SD) & $n=74$ & $n=151 \dagger$ & \\
\hline Mental component & $49.8(12.2)$ & $48.6(11.0)$ & 0.45 \\
\hline Physical component & $34.8(10.2)$ & $32.7 \quad(9.4)$ & 0.13 \\
\hline Diathesis, yr, mean (SD) & $12.3(15.1)$ & $10.6(12.9)$ & 0.53 \\
\hline
\end{tabular}


we used the nonparametric Mann-Whitney $U$ test to determine if differences in participants' characteristics between the pre-implementation and postimplementation study groups were significant. Calculations of the proportion of patients whose ulcers were healed at 3 months excluded those who had died or were lost to follow-up.

\section{Results}

Differences in patients admitted during the study periods before or after implementation of the new model of

Table 2: Clinical variables before and after implementation of the new model of care

\begin{tabular}{|c|c|c|c|}
\hline \multirow[b]{2}{*}{ Variable } & \multicolumn{2}{|c|}{ Care; no. $(\%)$ of patients } & \multirow[b]{2}{*}{$p$ value } \\
\hline & Old model & New model & \\
\hline Treatment frequency & $n=71$ & $n=167$ & \\
\hline None & $4 \quad(5.6)$ & - & $<0.001$ \\
\hline Once weekly & $3 \quad(4.2)$ & $34(20.8)$ & $<0.001$ \\
\hline Twice weekly & $16(22.5)$ & $83(49.7)$ & $<0.001$ \\
\hline 3 times per week & $21(29.6)$ & $40(24.0)$ & $<0.001$ \\
\hline Daily or more often & $27(38.0)$ & $10 \quad(6.0)$ & $<0.001$ \\
\hline $\begin{array}{l}\text { Use of compression } \\
\text { therapy, by cause of ulcer }\end{array}$ & $n=78$ & $n=180$ & \\
\hline Venous disease & $25 / 57(43.9)$ & 93/109 (85.3) & $<0.001$ \\
\hline Mixed disease & $7 / 21(33.3)$ & $44 / 71 \quad(62.0)$ & 0.014 \\
\hline $\begin{array}{l}\text { Healing rates, by cause } \\
\text { of ulcer }\end{array}$ & $n=78$ & $n=180$ & \\
\hline Venous disease & $13 / 57(22.8)$ & $64 / 109(58.7)$ & $<0.001$ \\
\hline Mixed disease & $5 / 21(23.8)$ & $36 / 71 \quad(50.7)$ & 0.029 \\
\hline Total & $18(23.1)$ & $100(55.6)$ & $<0.001$ \\
\hline
\end{tabular}

care were nonsignificant for sociodemographic variables, circumstance of living, HRQoL and clinical characteristics (Table 1).

Other between-period differences were seen once the new service was implemented, however, beginning with the plan of care. Under the new model, significantly fewer visits were planned after the initial assessment (Table 2); the proportion of daily visits, for example, dropped from $38 \%$ to $6 \%$ (Pearson $\chi^{2}$ test $60.1, p<0.001$ ). Overall, the proportion of patients treated with compression therapy increased significantly, as did 3-month healing rates. A larger proportion of patients with solely venous ulcers healed than did those with venous-arterial leg ulcers. Fig. 2 shows that healing rates improved (albeit with wide confidence intervals) toward the end of the pre-implementation study period, when the planning of the new service was well underway and nurse training had been completed. After implementation, the rate of patients whose leg ulcers were healed after 3 months was $48 \%-61 \%$, with tighter confidence intervals.

Differences between the study periods in reported SF12 scores at 3 months for the questionnaire's physical (mean 34.0 [standard deviation (SD) 4.5] v. 35.8 [SD 10.8], $t$ test $-1.6, p=0.12$ ) and mental components (mean 50.6 [SD 3.53] v. 50.7 [SD 11.0], $t$ test $-0.052, p=0.96$ ) were not significant. The mean physical component score for our study patients was lower than norms for all Canadians (50.5) as well as those older than 75 years (42.0), whereas the mean mental component score was closer to population norms (all adults, 51.7; those $>75$ years of age, 54.5 ). ${ }^{28}$

Comparing patients newly admitted during the last 6 months of either study period revealed the median number of nursing visits to have declined from 37 (interquartile

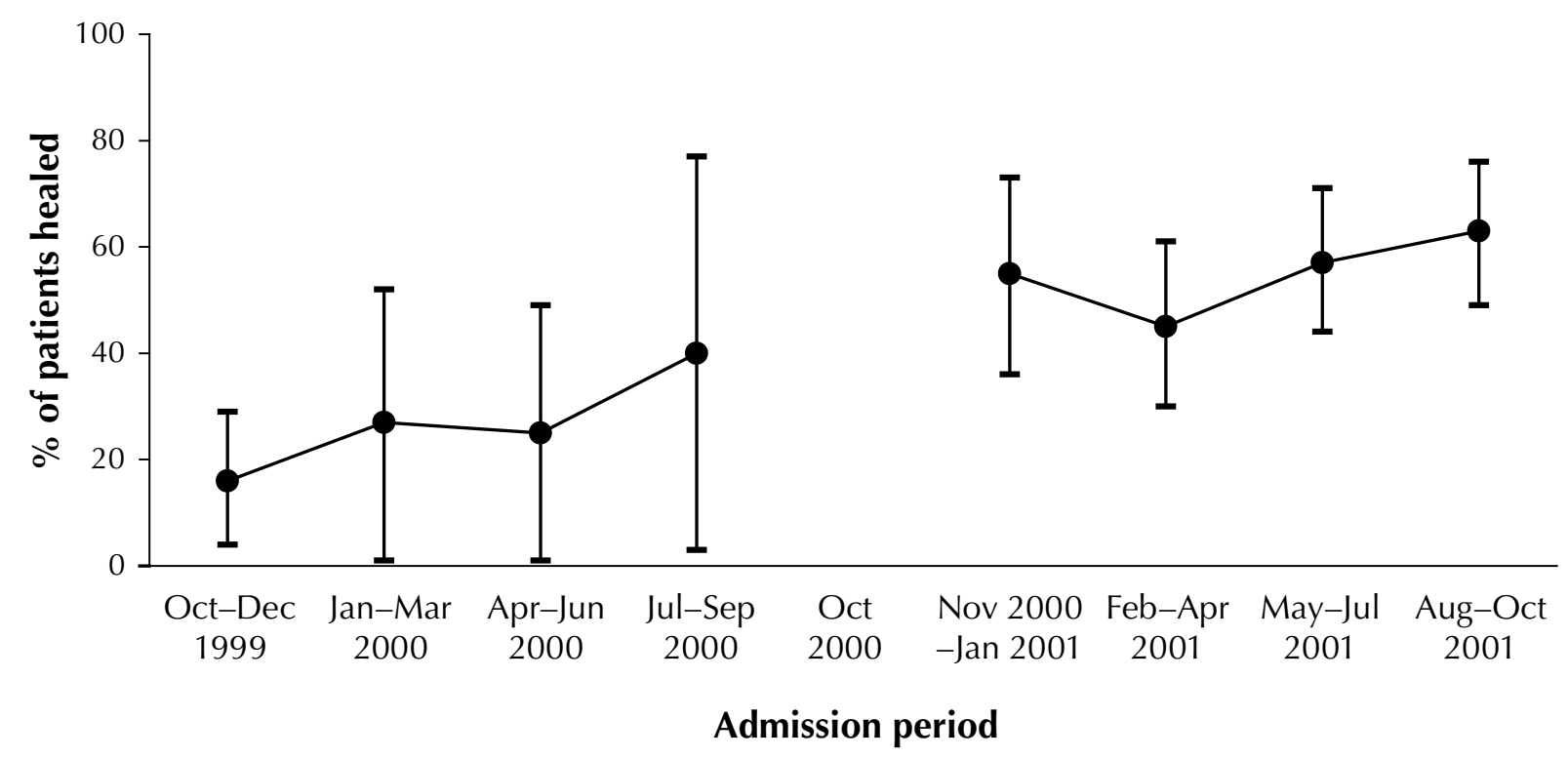

Fig. 2: Proportion of patients whose ulcers were healed within 3 months after admission, before and after implementation of the new service. Data were grouped quarterly, that is, by 3-month intervals within each 1-year study period. 
range [IQR] 24-42) to 25 (IQR 15-35) over the period ( $p=$ $0.041)$. The median number of visits to each patient declined from 3 (IQR 2-4.8) to 2.1 (IQR 1.6-2.4) per week $(p=0.005)$. The median supply cost per case decreased from \$1923 (IQR \$395-\$1931) to \$406 (IQR \$219-\$920; $p=0.005)$.

\section{Interpretation}

The purpose of this study was to evaluate an implementation of a new community approach to the treatment of leg ulcers, a common chronic wound. The fundamental aspect of the redesign was altering organizational structures to support the provision of guideline-driven practice. A dedicated team of registered nurses with additional training and appropriate team size ensured expertise and experience enough to provide quality care; the team was provided adequate financing, suitable equipment (e.g., hand-held Dopplers) and enhanced linkages to family physicians and specialists. The service was led by a clinical leader with combined clinical, teaching and management expertise. The implementation of this dedicated nurse-led service using up-to-date practice guidelines was accompanied by better healing rates and improved efficiencies. The proportion of patients receiving the appropriate treatment (compression bandaging) nearly doubled and the average number of care visits per person declined, yet healing rates nearly tripled. Given this, more patients could be provided care with the new model than with the old delivery model, for the same home-care budget overall.

Our conclusions are similar to those of Simon and colleagues ${ }^{29}$ who noted the cost-effectiveness and importance of nurses managing leg-ulcer care in the community in their comprehensive review. Our results were also similar to recent findings from a UK study where healing rates at 3 months more than doubled while the number of visits to provide care decreased..$^{30}$

Our study had several limitations. A prospective, observational "pre-post" evaluation, this study design cannot rule out secular trends that may have influenced the results. There is nevertheless good reason to attribute the improvement in 3-month healing rate and efficiencies to the way care was reorganized after implementation. An experimental study design would have been preferable, but it would have been a monumental task to convince several dozen health care authorities to allow their client populations to be randomly assigned. Another factor constraining the choice of study design was that the region was not prepared to delay the reorganization to plan a multi-site trial. Lastly, although it might be considered a limitation that the nurses providing care were involved in the collection of data, this was necessary to track healing in the community. Once bandages were applied, it would have been unduly intrusive (and prohibitively expensive) to remove them for an outcome assessment. On the other hand, our small team of dedicated, specially trained nurses ensured expert, quality outcome assessments. Consistency in data capture was enhanced by the use of standard clinical forms.

Guideline-driven care of this complex population of patients being treated in the community greatly improved health outcomes and efficiencies without infusion of new dollars. Leg ulcers are a chronic recurrent condition that requires specialized intervention, active self-management and proactive secondary prevention. This approach, which used population-based planning and guidelinedriven practice as the pillars of change, should be applied to other complex conditions and evaluated in other disease populations.

\section{This article has been peer reviewed.}

From the School of Nursing, Queen's University, Kingston, Ont. (Harrison, Friedberg); the School of Nursing (Graham), the Department of Medicine (Pierscianowski) and the Department of Surgery (Brandys), University of Ottawa; the Ottawa Health Research Institute, Clinical Epidemiology Program (Harrison, Friedberg, Graham); and the Victorian Order of Nurses (Lorimer), Ottawa, Ont.

Competing interests: Margaret Harrison and Ian Graham are Canadian Institutes of Health Research New Investigators. At the time the project was initiated, both were Ontario Ministry of Health and Long-Term Care Career Scientists, and their ministry start-up funds supported this study. An earlier version of this paper was presented at the Australian Concurrent National Symposia, Implementing Evidence Based Practice in Nursing and Midwifery, June 18, 2004.

Contributors: Margaret Harrison and Ian Graham were the principal investigators and co-wrote the first draft of the paper. Karen Lorimer was the leader of the Leg Ulcer Service, co-chaired the task force that developed the clinical protocol, and contributed to data acquisition. Elaine Friedberg conducted the statistical analysis. Tadeusz Pierscianowski (who also co-chaired the clinical protocol task force) and Tim Brandys were consulting physicians to the project and contributed to data acquisition. All of the authors were involved in the conceptualization and design of the study, participated in drafting or critically revising the manuscript, and approved the published version.

Acknowledgements: We thank the many patients with leg ulcers who agreed to participate in the study. We also acknowledge Val Angus for assistance in the preparation of this manuscript.

This research was possible through the ongoing support of the Ottawa Community Access Centre, Ottawa Victorian Order of Nurses, the Wound Service team in particular.

\section{References}

1. Graham ID, Harrison MB, Nelson EA, Lorimer K, Fisher A. A systematic review of prevalence studies of lower limb ulceration. Adv Skin Wound Care 2003;16:305-16.

2. Andersson E, Hansson C, Swanbeck G. Leg and foot ulcer prevalence and investigation of the peripheral arterial and venous circulation in a randomised elderly population. An epidemiological survey and clinical investigation. Acta Derm Venereol Suppl (Stockb) 1993;73:57-61.

3. Baker S, Stacy M, Jopp-McKay A, Thompson P. Epidemiology of chronic venous ulcers. Br 7 Surg 1991;64:258-61.

4. Callam MJ, Harper DR, Dale JJ, Ruckley CV. Chronic ulcer of the leg: clinical history. BM7 1987;294:1389-91.

5. Cornwall J, Dore C, Lewis J. Leg ulcers: Epidemiology and aetiology. $\operatorname{Br} 7$ Surg 1986;73:693-6.

6. Jull A, Walker A, Hackett M, Jones M, Rodgers A, Birchall N, et al. Leg ulceration and perceived health: a population base case-control study. Age Ageing 2004;33:236-41.

7. Franks P, McCullagh L, Moffatt CJ. Assessing quality of life with chronic venous leg ulceration using the Medical Outcomes Short Form-36 questionnaire. Ostomy Wound Manage 2003;49:26-37.

8. Walters S, Morrel C, Dixon S. Measuring health-related quality of life in patients with venous leg ulcers. Qual Life Res 1999;8:325-36.

9. Korn P, Patel S, Heller J, Deitch J, Krishnasastry J, Bush H, et al. Why insurers should reimburse patients for compression stocking in patients with chronic venous stasis. 7 Vasc Surg 2002;35:950-57.

10. Laing W. Chronic venous diseases of the leg. Report \#108. 1992. London, UK (SW1A 2DY): Office of the Health Economics. 
11. Harrison MB, Graham ID, Friedberg E, Lorimer K, Vandevelde-Coke S; Ottawa-Carleton Regional Leg Ulcer Project. Assessing the population with leg and foot ulcers. Can Nurse 2001;97:18-23.

12. Friedberg E, Harrison MB, Graham ID. Current home care expenditures for persons with leg ulcers. 7 Wound Ostomy Continence Nurs 2002;29:186-92.

13. Graham ID, Harrison MB, Shafey M, Keast D. Knowledge and attitudes regarding care of leg ulcers: survey of family physicians. Can Fam Physician 2003;49:896-902.

14. Graham ID, Harrison MB, Moffat C, Franks P. Leg ulcer care: nursing attitudes and knowledge. Can Nurse 2001;97:19-24.

15. Cullum N, Nelson EA, Fletcher A, Sheldon T. Compression for venous leg ulcers. Cochrane Database of Systematic Reviews 2004;(2):CD 000265.

16. Nelson EA, Cullum N, Jones J. Venous leg ulcers. In: Jones G, editor. Clinical evidence. London: BMJ Publishing, 2002. p. 2031-45.

17. Lorimer K, Harrison MB, Graham ID, Friedberg E, Davies B. Venous leg ulcer care: How evidence based is nursing practice? 7 Wound Ostomy Continence Nurs 2003;30:132-42.

18. Lorimer K. Continuity through best practice: design and implementation of a nurse-led community leg ulcer service. Can 7 Nurs Res 2004;36:3-6.

19. Graham ID, Lorimer K, Harrison MB, Pierscianowski T, for the Leg Ulcer Protocol Tasks Force, Leg Ulcer Protocol Task Force Working Group, et al. Evaluating the quality and content of international clinical practice guidelines for leg ulcers: preparing for Canadian adaptation. Can Assoc Enterostomal Ther 7 2000;19(3):15-31.

20. Graham ID, Harrison MB, Lorimer K, Piercianowski T, Friedberg E, Buchanan $M$, et al. Advances in skin and wound care, adapting national and international leg ulcer practice guidelines for local use: the Ontario Leg Ulcer Community Care Protocol. Adv Skin Wound Care 2005 (in press).

21. Manthey M. A theoretical framework for primary nursing. 7 Nurs Adm 1980; $10: 11-5$
22. Graham ID, Harrison MB, Brouwers M, Davies B, Dunn S. Facilitating the use of evidence in practice: evaluating and adapting clinical practice guidelines for local use by health care organizations. 7 Obstet Gynecol Neonatal Nurs 2002;31:475-87.

23. Graham ID, Harrison MB, Brouwers M. Evaluating and adapting practice guidelines for local use: a conceptual framework. In: Pickering S, Thompson J, editors. Clinical governance in practice. London: Harcourt, 2003. p. 213-229.

24. Melzack R. The short-form McGill Pain Questionnaire. Pain 1987;30:191-7.

25. Ware JE, Kosinski M, Keller SD. A 12-item short-form health survey - construction of scales and preliminary tests of reliability and validity. Med Care 1996;34:220-33.

26. Margolis D, Allen-Taylor L, Hoffstad O, Berlin J. The accuracy of venous leg ulcer prognostic models in a wound care system. Wound Repair Regen 2004;12 $163-68$

27. Margolis DJ, Berlin JA, Strom BL. Which venous leg ulcers will heal with limb compression bandages? Am 7 Med 2000;109:15-9.

28. Hopman W, Towheed T, Anastassiades T, Tenenhouse A, Poliquin S, Berger $\mathrm{C}$, et al. Canadian normative data for the SF-36 health survey. CMAf 2000; $163: 265-71$.

29. Simon D, Dix F, McCollum C. Management of venous leg ulcers. BM7 2004 328:1358-62

30. Moffatt CJ, Franks P. Implementation of a leg ulcer strategy. $\operatorname{Br} \mathcal{F}$ Dermatol 2004;151:857-67.

Correspondence to: Margaret B. Harrison, School of Nursing, Queen's University, 78 Barrie St., Kingston ON K7L 3N6;

fax 613 533-6331; harrisnm@post.queensu.ca
A five level credit program exclusively for physicians designed to develop superior leadership and management skills

Approved for RCPSC, CFPC, CCHSE credits

\section{In-house PMI}

A practical, cost effective and focused training opportunity held on-site for medical leaders and managers

For information:

tel $800663-7336$ or 613 731-8610 X2319 (PMI) or X2261 (In-house PMI) professional_development@cma.ca

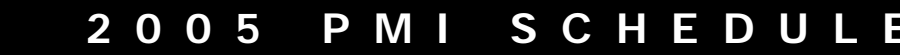

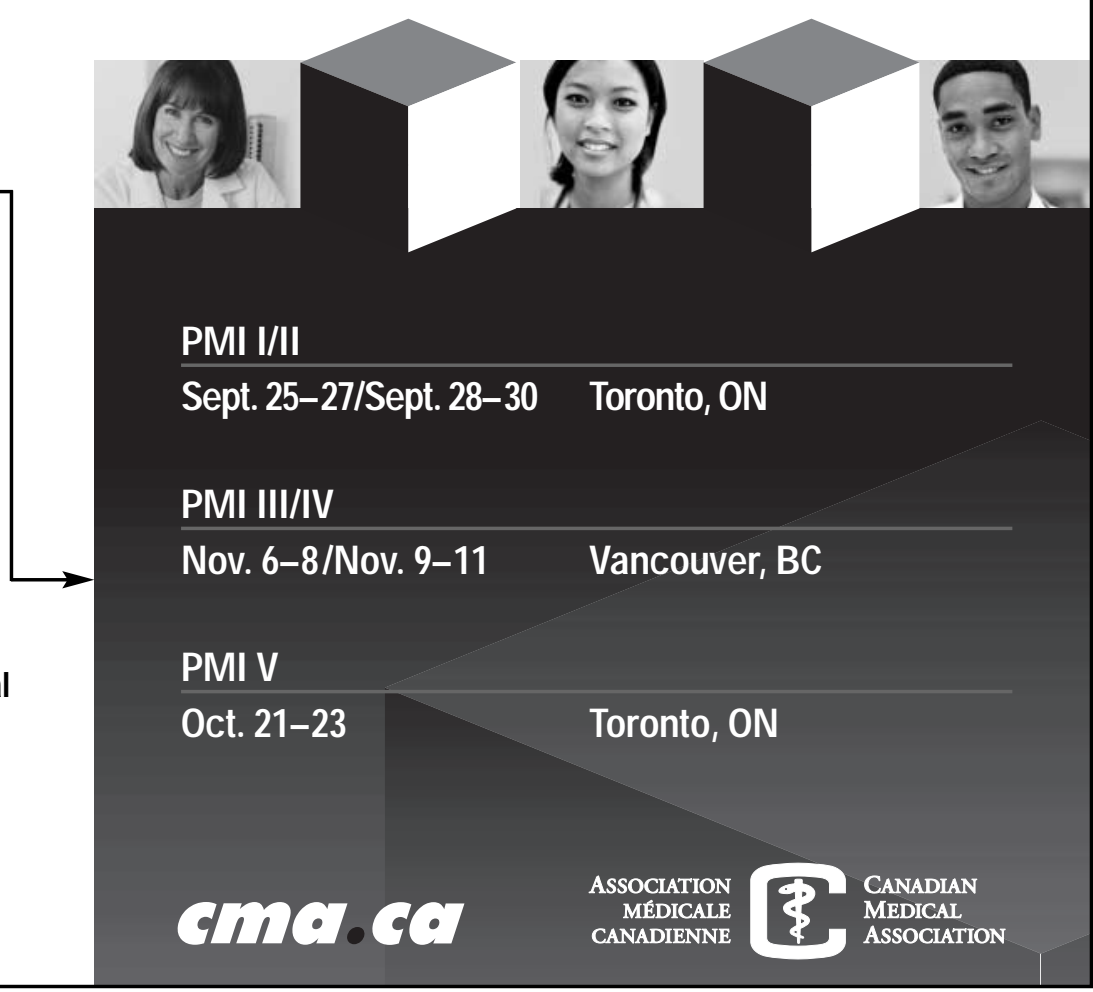

